\title{
Thickness effect on the structure, grain size, and local piezoresponse of self-polarized lead lanthanum zirconate titanate thin films
}

Cite as: J. Appl. Phys. 120, 054101 (2016); https://doi.org/10.1063/1.4960137

Submitted: 02 June 2016 . Accepted: 19 July 2016 . Published Online: 02 August 2016

M. Melo, E. B. Araújo, V. V. Shvartsman, V. Ya. Shur (D), and A. L. Kholkin (D)

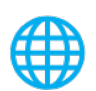

View Online

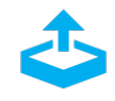

\section{ARTICLES YOU MAY BE INTERESTED IN}

Thickness dependence of structure and piezoelectric properties at nanoscale of polycrystalline lead zirconate titanate thin films

Journal of Applied Physics 113, 187206 (2013); https://doi.org/10.1063/1.4801961

Ferroelectric thin films: Review of materials, properties, and applications

Journal of Applied Physics 100, 051606 (2006); https://doi.org/10.1063/1.2336999

Ferroelectric, pyroelectric, and piezoelectric properties of a photovoltaic perovskite oxide Applied Physics Letters 110, 063903 (2017); https://doi.org/10.1063/1.4974735

\section{Applied Physics Reviews} Now accepting original research

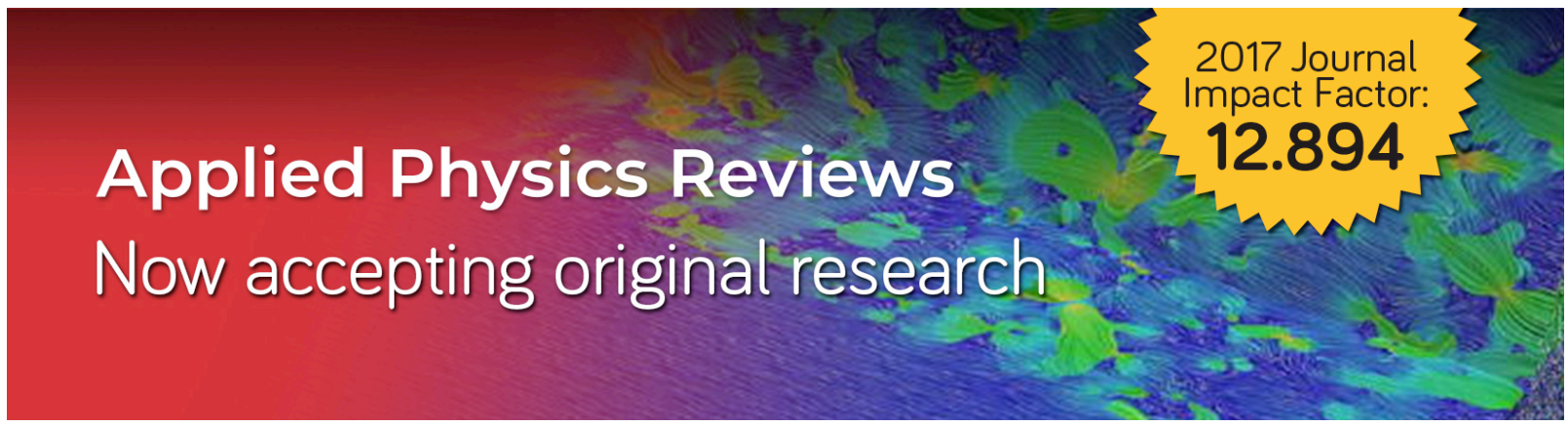




\title{
Thickness effect on the structure, grain size, and local piezoresponse of self-polarized lead lanthanum zirconate titanate thin films
}

\author{
M. Melo, ${ }^{1}$ E. B. Araújo, ${ }^{1, a)}$ V. V. Shvartsman, ${ }^{2}$ V. Ya. Shur, ${ }^{3}$ and A. L. Kholkin ${ }^{3,4}$ \\ ${ }^{1}$ Departamento de Física e Química, Faculdade de Engenharia de Ilha Solteira, UNESP-Univ. Estadual \\ Paulista, 15385-000 Ilha Solteira, SP, Brazil \\ ${ }^{2}$ Institute for Materials Science, University Duisburg-Essen, 45141 Essen, Germany \\ ${ }^{3}$ Institute of Natural Sciences, Ural Federal University, 620000 Ekaterinburg, Russia \\ ${ }^{4}$ Department of Physics and CICECO_Aveiro Institute of Materials, University of Aveiro, 3810-193 Aveiro, \\ Portugal
}

(Received 2 June 2016; accepted 19 July 2016; published online 2 August 2016)

\begin{abstract}
Polycrystalline lanthanum lead zirconate titanate (PLZT) thin films were deposited on $\mathrm{Pt} / \mathrm{TiO}_{2} / \mathrm{SiO}_{2} /$ Si substrates to study the effects of the thickness and grain size on their structural and piezoresponse properties at nanoscale. Thinner PLZT films show a slight (100)-orientation tendency that tends to random orientation for the thicker film, while microstrain and crystallite size increases almost linearly with increasing thickness. Piezoresponse force microscopy and autocorrelation function technique were used to demonstrate the existence of local self-polarization effect and to study the thickness dependence of correlation length. The obtained results ruled out the bulk mechanisms and suggest that Schottky barriers near the film-substrate are likely responsible for a build-in electric field in the films. Larger correlation length evidence that this build-in field increases the number of coexisting polarization directions in larger grains leading to an alignment of macrodomains in thinner films. Published by AIP Publishing. [http://dx.doi.org/10.1063/1.4960137]
\end{abstract}

\section{INTRODUCTION}

Recent advances in microelectronic technology and the continued demand for portability in consumer electronics has stimulated intensive research on the fundamental properties of ferroelectric thin films. Sometimes, the physical properties of polycrystalline thin films are very different with respect to bulk materials, since the ferroelectricity phenomenon exhibits an intrinsic dependence on sample size because a different degree of ordering occurs near surfaces or interfaces. After the advent of new instrumentation to probe these physical properties with a nanoscale resolution and motivated by problems associated with downscaling and socalled extrinsic contributions in ferroelectrics, the physical properties of ferroelectric thin films are periodically revisited to better understand the basic physics of such size reduction providing possibilities to develop high performance devices.

La-modified lead zirconate titanate, $\mathrm{Pb}_{1-x} \mathrm{La}_{x}$ $\left(\mathrm{Zr}_{1-y} \mathrm{Ti}_{y}\right)_{1-x / 4} \mathrm{O}_{3}$ (PLZT), is a ferroelectric material known to exhibit remarkable dielectric, piezoelectric, pyroelectric, and electrooptic properties with potential for technological applications. ${ }^{1,2}$ Depending on the $\mathrm{La} / \mathrm{Zr} / \mathrm{Ti}$ composition, PLZT exhibits a relaxor behavior, giving rise to very large dielectric permittivity and electro-optical coefficients. ${ }^{3}$ The relaxor behavior is related to the charge disorder caused by cations of different valences randomly distributed over the equivalent crystallographic positions, i.e., $\mathrm{Pb}^{2+}$ and $\mathrm{La}^{3+}$ on A sites of the perovskite structure of PLZT. The composition $\mathrm{La} / \mathrm{Zr} / \mathrm{Ti}=9 / 65 / 35 \mathrm{~mol}$. \% is particularly important because most of the properties of technological interest show their

\footnotetext{
a) Author to whom correspondence should be addressed. Electronic mail: eudes@dfq.feis.unesp.br
}

maximum values at this ratio. ${ }^{4}$ In the thin film form, the great potential of the PLZT has been demonstrated over past years for applications in waveguides, ${ }^{5}$ capacitors, ${ }^{6}$ photovoltaic ultraviolet sensors, ${ }^{7}$ and others.

In the present work, we investigate the effects of thickness and grain size on structural properties, local piezoelectric properties, and correlation length of polycrystalline PLZT thin films prepared by chemical route. The structural properties were investigated using X-ray diffraction (XRD) technique at room temperature, while the piezoresponse force microscopy (PFM) technique has been used to study the piezoelectric properties at nanoscale. The main contribution of the present work is to provide a qualitative relationship between grain size and the size of nanodomains in the studied PLZT films.

\section{EXPERIMENTAL}

PLZT thin films with a nominal composition of $\left(\mathrm{Pb}_{0.91} \mathrm{La}_{0.09}\right)\left(\mathrm{Zr}_{0.65} \mathrm{Ti}_{0.35}\right)_{0.977} \mathrm{O}_{3}$ were deposited on $\mathrm{Pt} / \mathrm{TiO}_{2} /$ $\mathrm{SiO}_{2} / \mathrm{Si}(100)$ substrates using a chemical route based on modified Pechini method as described elsewhere. ${ }^{8}$ A polymeric resin with metallic ions randomly distributed along polymeric chain has been used to prepare films by spin coating at $5000 \mathrm{rpm}$ for $30 \mathrm{~s}$. Pyrolysis was carried out in air by putting the deposited films directly on a hot-plate at $\sim 200^{\circ} \mathrm{C}$ for $5 \mathrm{~min}$ and then in an electric furnace at $300^{\circ} \mathrm{C}$ for $1 \mathrm{~h}$. The film thickness increases when depositing a new layer on the previously pyrolyzed film layer and repeating the same procedure to remove the organics. Upon obtaining the desired film thickness, a final temperature annealing at $700^{\circ} \mathrm{C}$ for $1 \mathrm{~h}$ promotes the final film crystallization. The obtained films were $240,350,430$, and $540 \mathrm{~nm}$ in thickness. 
X-ray diffraction (XRD) has been used to study the structural properties of the PLZT films using a Rigaku Ultima IV diffractometer with $\mathrm{CuK}_{\alpha}(1.5406 \AA)$ radiation. The structural parameters were obtained from Rietveld refinements. For the Rietveld analysis, ${ }^{9}$ XRD data were employed using EXPGUI interface. ${ }^{10}$ The peak profiles have been fitted by using the Thompson-Cox-Hastings ${ }^{11}$ pseudo-Voigt function while a sixth-order polynomial has been used to fit the background. An orthorhombic phase with Prmm space group has been considered as the initial configuration for the refinements, where the $\mathrm{Pb}^{2+}$ and $\mathrm{La}^{2+}$ ions occupy sites at $(0,0,0), \mathrm{Ti}^{4+}$ / $\mathrm{Zr}^{4+}$ and $O_{I}^{2-}$ occupy sites $(1 / 2,1 / 2, \mathrm{z})$, and $O_{I I}^{2-}$ occupy sites at $(1 / 2,0, \mathrm{z})$. The goodness-of-fit $(\mathrm{GOF}) \chi^{2}=\left(R_{w p} / R_{\text {exp }}\right)^{2}$ attests the quality of a fit, where $R_{\mathrm{wp}}$ and $R_{\exp }$ indices are the weighted profile and the statistically expected R-factors, respectively. ${ }^{12}$ The refined parameters include background, scale factor, zero correction, peak width, cell parameters, positional coordinates, and isotropic thermal parameters. The use of anisotropic thermal parameters was also experimented for $\mathrm{Ti}^{4+} / \mathrm{Zr}^{4+}, \mathrm{O}_{I}^{2-}$, and $O_{I I}^{2-}$ ions, but no improvement in the agreement factors is observed. Based on the refined data, Williamson-Hall (WH) analysis was applied to evaluate the microstrain $(\Delta d / d)$ and crystallite size (D) of the PLZT films according to $\Gamma \cos \theta=\lambda / D+(\Delta d / d) 4 \sin \theta$, where $d$ is the lattice spacing, $\Gamma$ is the full width at half maximum (FWHM) value due to peak broadening, $\lambda$ is the wavelength, and $\theta$ is the Bragg angle.

Polar structures on the surface of the films were studied by piezoresponse force microscopy (PFM) technique using a commercial AFM (Ntegra Prima, NT-MDT) equipped with a tip-cantilever system (Nanosensors) having a spring constant of $42 \mathrm{~N} / \mathrm{m}$ and a tip apex radius less than $10 \mathrm{~nm}$. The PFM images were acquired under a probing $\mathrm{AC}$ voltage with amplitude of $5 \mathrm{~V}$ and frequency of $50 \mathrm{kHz}$. The driving frequency was chosen being far from contact resonance in the cantilever-sample system to avoid the ambiguity experimental data. The PFM signal was acquired by a lock-in amplifier (SR-830 A, Stanford Research) set for the time constant and sensitivity of $10 \mathrm{~ms}$ and $1 \mathrm{mV}$, respectively. Domain images ( $\mathrm{x}=\mathrm{R} \cos \varphi$, where $\mathrm{R}$ is the amplitude, and $\varphi$ is the phase of piezoresponse) were obtained both in both lateral (lateral PFM-LPFM) and vertical (vertical PFM-VPFM) modes. ${ }^{13}$ In this way, bright and dark contrasts on the PFM images reflect the parallel or antiparallel polarization directions relative to the probing electric field, respectively.

\section{RESULTS AND DISCUSSION}

Fig. 1 shows the observed, calculated, and difference profiles obtained by Rietveld refinements of the XRD data for polycrystalline randomly oriented PLZT thin films of different thicknesses. The indexed (hkl) peaks in Fig. 1 refer to the orthorhombic phase of the PLZT with Pmmm space group. The peaks $\operatorname{Pt}(111)$ and $\operatorname{Pt}(200)$ refer to the cubic phase of the platinum substrate with the space group $F m \overline{3} m$ and lattice parameter $a=3.892 \AA$. An agreement between the observed and calculated patterns in this figure is indicative of a good fit. The positional coordinates from refinements for the thicker and thinner PLZT films are summarized in Table I while the

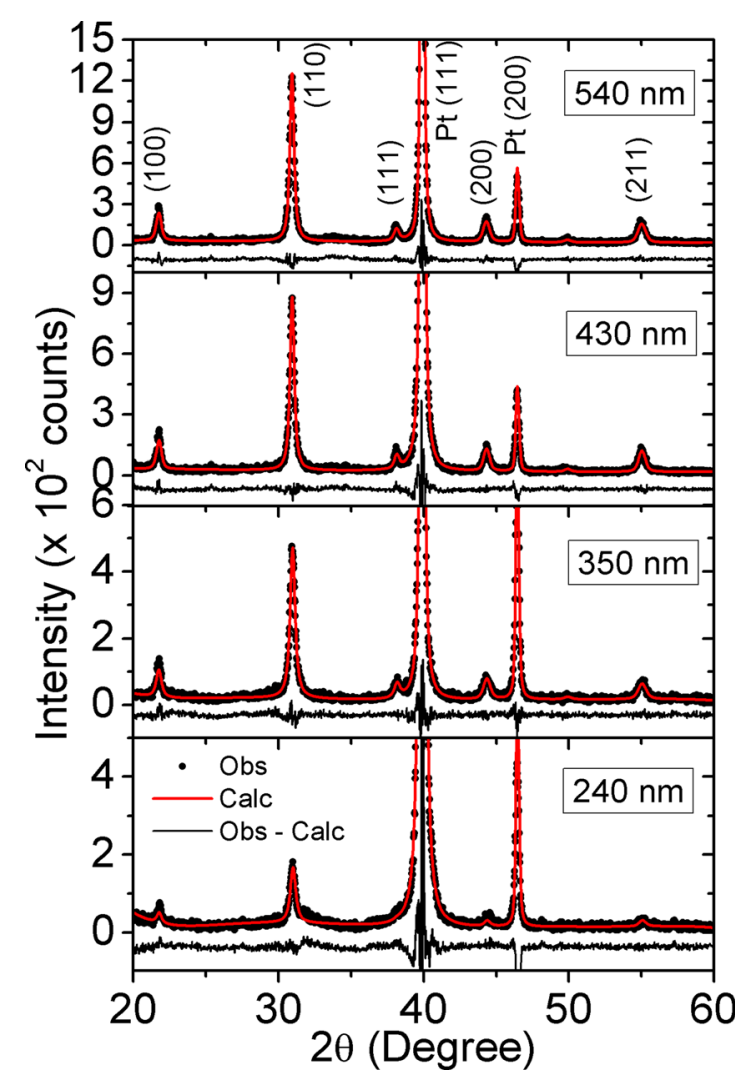

FIG. 1. Observed (dots), calculated (lines), and difference (bottom lines) XRD profiles of PLZT thin films at different thicknesses.

lattice parameters, volume cell, crystallographic R-factors, and goodness-of fit indices for different thicknesses are given in Table II. These lattice parameters shown in Table II are in good agreement with those observed for PLZT bulk ceramics for the same composition. ${ }^{14}$ In principle, small $\chi^{2}$ factor for all data in Table II is an indicative of good refinements. However, numerically, we must also consult $R_{p}$ and $R_{w p}$ indices to judge on the quality of the fit. $R_{p}$ quantifies the difference between the observed and calculated data while $R_{\mathrm{wp}}$ weights the residual so that higher intensity data points are more representative than low intensity data points. Thus, $R_{w p}$ can be unfavorable in situations such as the important information is contained in the weakest peaks. This is not the case in the present work. For Rietveld analysis on single crystals or ceramic data, R-factors are expected to be less than $10 \%$. However, data noise and peaks width larger in the

TABLE I. Refined structural parameters for the thinner and thicker PLZT films.

\begin{tabular}{|c|c|c|c|c|c|c|c|c|}
\hline \multirow[b]{2}{*}{ Atom } & \multicolumn{4}{|c|}{ Thickness $240 \mathrm{~nm}$} & \multicolumn{4}{|c|}{ Thickness $540 \mathrm{~nm}$} \\
\hline & $\mathrm{X}$ & $\mathrm{Y}$ & $\mathrm{Z}$ & $\mathrm{U}_{\text {iso }}\left(\AA^{2}\right)$ & $\mathrm{X}$ & $\mathrm{Y}$ & $\mathrm{Z}$ & $\mathrm{U}_{\text {iso }}\left(\AA^{2}\right)$ \\
\hline $\mathrm{Pb}^{2+}$ & 0.0000 & 0.0000 & 0.0000 & 0.1459 & 0.0000 & 0.0000 & 0.0000 & 0.1061 \\
\hline $\mathrm{La}^{2+}$ & 0.0000 & 0.0000 & 0.0000 & 0.2320 & 0.0000 & 0.0000 & 0.0000 & 0.8000 \\
\hline $\mathrm{Ti}^{4+}$ & 0.5228 & 0.5228 & 0.5228 & 0.0369 & 0.4670 & 0.4670 & 0.4670 & 0.6343 \\
\hline $\mathrm{Zr}^{4+}$ & 0.5228 & 0.5228 & 0.5228 & 0.0474 & 0.4647 & 0.4646 & 0.4646 & 0.0400 \\
\hline $\mathrm{O}_{\mathrm{I}}^{2-}$ & 0.5200 & 0.5250 & 0.0350 & 0.8000 & 0.5199 & 0.5249 & 0.0349 & 0.5540 \\
\hline $\mathrm{O}_{\mathrm{II}}^{2-}$ & 0.5334 & 0.0404 & 0.5344 & 0.7606 & 0.5333 & 0.0403 & 0.5343 & 0.3900 \\
\hline $\mathrm{O}_{\text {III }}^{2-}$ & 0.0394 & 0.5363 & 0.5374 & 0.7741 & 0.1051 & 0.5413 & 0.5226 & 0.2187 \\
\hline
\end{tabular}


TABLE II. Summary structural parameters and R-factors from Rietveld refinements of PLZT films with different thicknesses.

\begin{tabular}{|c|c|c|c|c|c|c|c|c|}
\hline \multirow[b]{2}{*}{ Thickness } & \multicolumn{4}{|c|}{ Lattice parameters and volume cell } & \multicolumn{4}{|c|}{$\mathrm{R}$-factors and $\mathrm{GOF}$} \\
\hline & $a(\AA)$ & $b(\AA)$ & $c(\AA)$ & $V\left(\AA^{3}\right)$ & $\mathrm{R}_{\mathrm{wp}}$ & $\mathrm{R}_{\mathrm{p}}$ & $\mathrm{R}_{\exp }$ & $\chi^{2}$ \\
\hline $540 \mathrm{~nm}$ & $4.090(4)$ & $4.096(9)$ & $4.086(3)$ & $68.48(5)$ & 26.20 & 16.12 & 21.81 & 1.44 \\
\hline $430 \mathrm{~nm}$ & $4.085(4)$ & $4.098(0)$ & $4.088(3)$ & $68.44(6)$ & 23.35 & 13.25 & 19.17 & 1.48 \\
\hline $350 \mathrm{~nm}$ & $4.097(2)$ & $4.094(5)$ & $4.089(3)$ & $68.60(4)$ & 12.15 & 7.06 & 10.75 & 1.29 \\
\hline $240 \mathrm{~nm}$ & $4.095(6)$ & $4.095(6)$ & $4.090(6)$ & $68.59(2)$ & 13.24 & 7.12 & 8.44 & 2.46 \\
\hline
\end{tabular}

XRD patterns of thin films result in higher $R_{w p}, R_{p}$, and $R_{\exp }$ values. Therefore, although $\mathrm{R}$-factors are larger in the present work, small $\chi^{2}$ values and a small difference between observed and calculated data as shown Fig. 1 are indicative of good refinements. Consequently, the structural parameters and calculated lattice parameters summarized in Tables I and II are representative values for the studied films in the present work.

From refined parameters, the microstrain $(\Delta d / d)$ and the crystallite size (D) evaluated using Williamson-Hall analysis are plotted in Figs. 2(a) and 2(b), respectively, as a function of the film thickness. As seen, both microstrain and crystallite size increases with increasing film thickness. The obtained microstrain $(0.17 \%-0.39 \%)$ in this work for polycrystalline PLZT films are comparable to the values reported in literature for (100)-oriented PZT thin films prepared by rfsputtering technique. ${ }^{15}$ The observed thickness dependence of the microstrain can be a direct effect of the polymeric chemical method used to prepare the films. This result suggests that thinner films reduce the interplanar spacing minimizing the stacking fault in the films. On the other hand, several depositions increase the film thickness but irregularities in the planar stacking also increase. The obtained

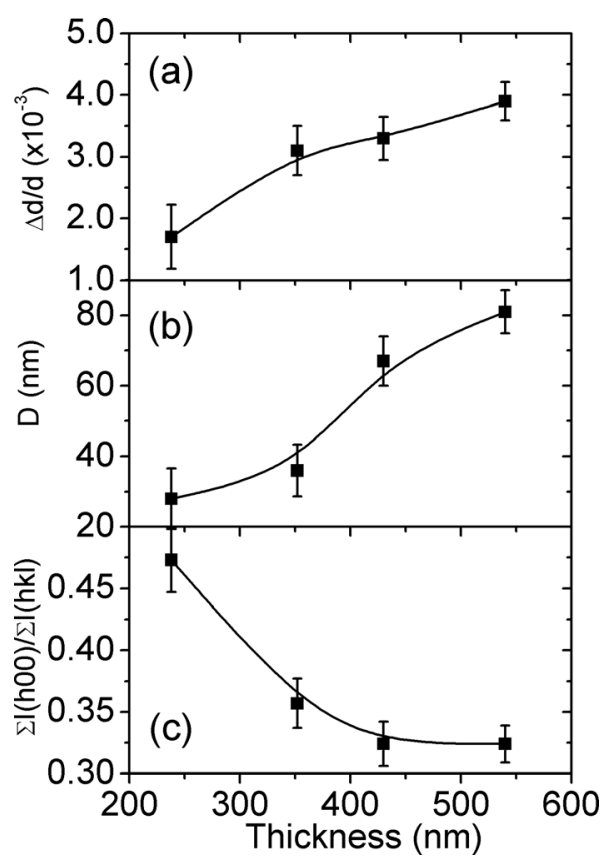

FIG. 2. (a) Microstrain, (b) crystallite size, and (c) orientation ratio of PLZT thin films as a function of film thickness. Lines are drawn as a guide to the eye. crystallite size values $(28-81 \mathrm{~nm})$ were also close to values reported in those PZT films. The observed increase of crystallite size with increasing film thickness is in line with several reports on ferroelectric films.

The thickness dependence of the orientation factor $\alpha=\sum(h 00) / \sum(h k l)$ is shown in Fig. 2(c). The $\alpha$ ratio was 0.32 for the film of $540 \mathrm{~nm}$ in thickness and increased to 0.47 when the film thickness decreased to $240 \mathrm{~nm}$. This result demonstrates a slight (100)-orientation tendency for thinner PLZT films while thicker films show a random orientation behavior.

Some works in literature have discussed the mechanisms behind the preferred orientations in PLZT thin films prepared by different routes. ${ }^{16,17}$ For rhombohedral films prepared by sol-gel method, these have been attributed the (100)-orientation of PLZT thin films on Pt substrates to the presence of a $\mathrm{PbO}$ buffer layer while the (111)-orientation have been mainly associated with the lattice matching between filmsubstrate or to the effect an inter-metallic phase $\mathrm{Pt}_{5-7} \mathrm{~Pb}$ in the early stage of the crystallization. ${ }^{18}$ In addition, the (111)preferred orientations for tetragonal PLZT films prepared by rf-sputtering have been attributed to lattice mismatch with the (111)-oriented platinum substrate. ${ }^{17}$ In the present work, there was no indication of the lattice matching between film and the substrate or of the presence of an additional intermediate phase between film-substrate leading to observed small (100)-orientation tendency. Probably, the growth tendency observed in the present work for thinner films relaxes at thicker films after multiple depositions.

The topography and vertical piezoresponse images of PLZT thin films at different thicknesses are shown in Figs. 3(a) and 3(b), respectively. The topography of the studied films is very similar, except that for the thicker film exhibiting smaller grains as observed in Fig. 3(a). The bright and dark areas in out-of-plane images in Fig. 3(b) refer to upward and downward polarization states, respectively, while intermediate contrast refers to the weak piezoresponse VPFM signal. Fig. 3(b) reveals that the studied films are quite homogeneous in terms of piezoresponse.

From topography images in Fig. 3(a), we measured the grain sizes. Then, the measured average grain sizes were plotted as a function of film thickness in Fig. 4(a). It was observed that the film with $350 \mathrm{~nm}$ in thickness has larger grain size $(738 \mathrm{~nm})$ while the thicker film $(540 \mathrm{~nm}$ in thickness) presents smaller grain size $(438 \mathrm{~nm})$. The procedure employed for measuring the grain size considered spherical grains, although some elongated grains were observed. In contrast to the topography observed in the present work for randomly oriented films, triangular grains reported in literature for PLZT films prepared by rf-sputtering are associated with the preferential (111) orientation in the films. ${ }^{18}$ Excluding the texture effects on the morphology of our films (these should be indeed minimal for the top layer), a large amount per deposition of material to be crystallized may lead to spherical grains observed in the present work.

Fig. 4(b) shows the piezohistograms of PLZT films acquired from piezoelectric out-of-plane images in Fig. 3(b) at different thicknesses. The piezohistograms are statistical distributions of the piezoelectric signal related to the domain 
(a)

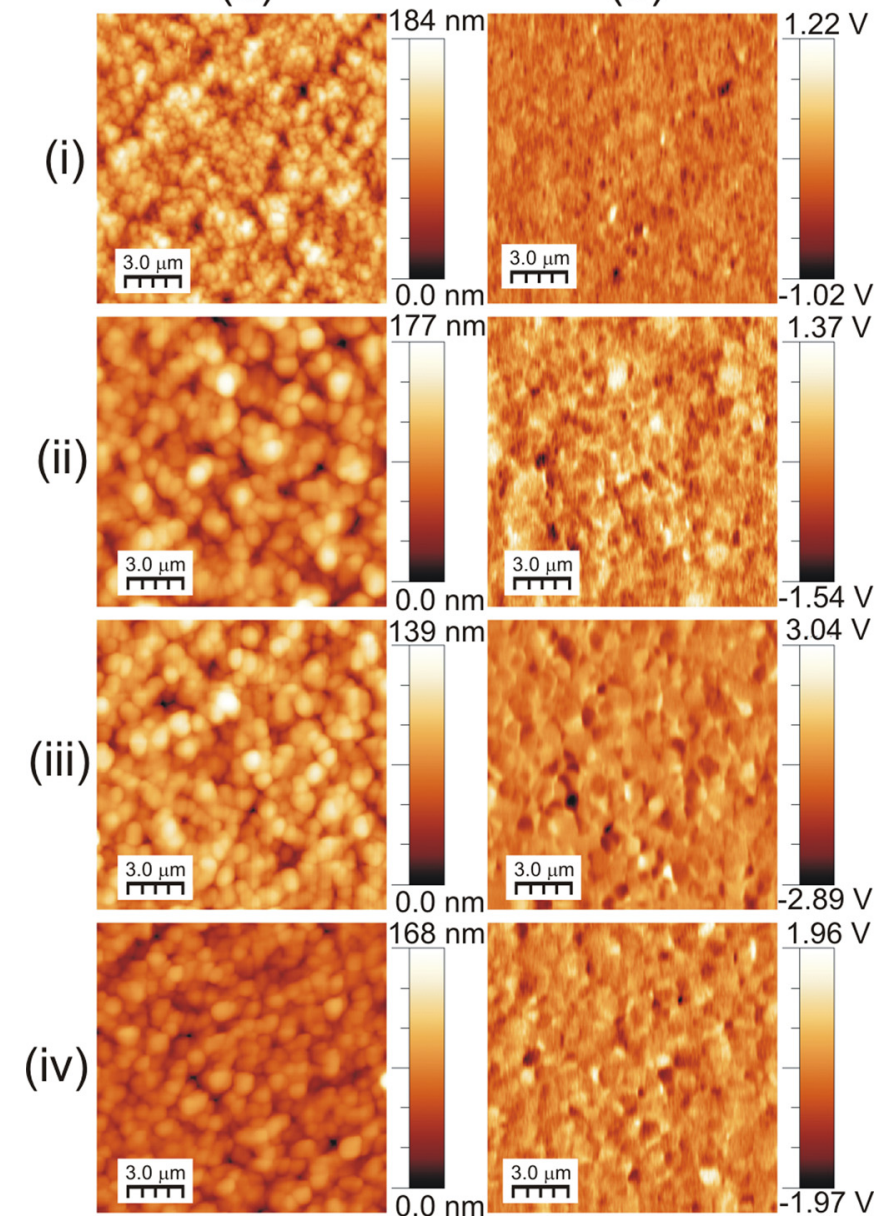

FIG. 3. (a) Topography and (b) VPFM piezoresponse images $\left(15 \times 15 \mu \mathrm{m}^{2}\right)$ of PLZT thin films for the thicknesses: $540 \mathrm{~nm}$ (i), $430 \mathrm{~nm}$ (ii), $350 \mathrm{~nm}$ (iii), and $240 \mathrm{~nm}$ (iv).

configuration in a ferroelectric, ${ }^{19}$ such as for out-of-plane polarization the vertical tip displacement is due to the effective $d_{33}$ piezoelectric coefficient. ${ }^{13}$ Thus, the observed peaks in these distribution curves are associated with the most probable domain configuration while the peak width is a measure of a number of domain states. These piezohistograms distribution in Fig. 4(b) suggests single peaks for films with 240,430 , and $540 \mathrm{~nm}$ in thickness, but the distribution of the piezoresponse signal for the film with $350 \mathrm{~nm}$ in thickness clearly exhibits a peak close to $0 \mathrm{~V}$ and a shoulder around -1 V. Lines in Fig. 4(b) refer to fit curves performed by using Gaussian functions for piezohistograms deconvolution. Only one peak for films with 240,430 , and $540 \mathrm{~nm}$ in thickness indicates predominance of unimodal domains, and, for this reason, one Gaussian function has been used to fit the respective piezohistograms. On the other hand, the bimodal distribution of the film with $350 \mathrm{~nm}$ thick suggest a predominance of domains centered around $0 \mathrm{~V}$ with another active piezoregion, or "dynamic" polar nanoregion, ${ }^{13}$ shifted to negative voltages $(\sim 1.0 \mathrm{~V})$. Then, two Gaussian functions were used to fit this piezohistogram. Despite two Gaussians used in our analyses, we consider the linewidth of the main curve around $0 \mathrm{~V}$, since the secondary peak around $-1 \mathrm{~V}$ is too small and its linewidth is not considered. However, the
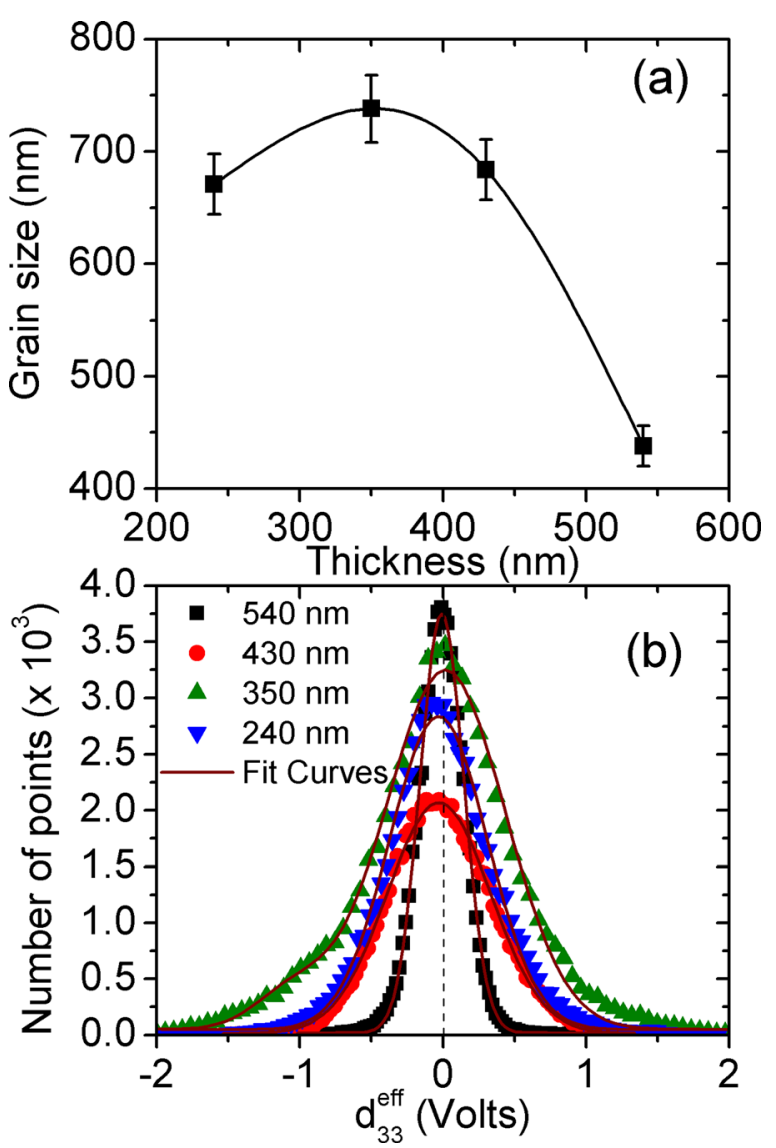

FIG. 4. (a) Thickness dependence of average grain size of studied PLZT thin films. (b) Out-of-plane distribution of PLZT thin films at different thicknesses. Line in (a) is drawn as a guide to the eye while lines in (b) refers to fit curves.

presence of this secondary peak affects the linewidth of the main peak, as expected, and for this reason, we consider the linewidth of the main peak for the film with $350 \mathrm{~nm}$ thick.

Important information can be obtained from curves fitting in Fig. 4(b). The half-width of peaks of piezohistograms are important parameter to be considered, as information can be obtained to infer about the polarization directions evolution. In polycrystalline thin films with random orientation, the half-width broadening can indicate various polarization directions in coexistence. ${ }^{13}$ The thickness dependence of the peaks half-width in piezohistograms from Fig. 4(b) is shown in Fig. 5(a). Looking more closely to the curve shapes in Figs. 4(a) and 5(a), we observe that both curves show essentially the same shape with a maximum at the film with $350 \mathrm{~nm}$ in thickness as well a minimum at the thicker film $(540 \mathrm{~nm}$ thick). These results suggest an intrinsic relationship between the coexisting polarization directions and the grain size. In other words, the coexisting polarization directions increase with increasing grain size in the studied films. This assumption agrees with the observed thickness dependence of crystallite size in Fig. 2(b). As the domain orientation inside the grains depends on the internal crystalline structure and larger grain sizes may host a large number of smaller crystallites, we expect a more complex internal crystalline structure for thinner films having larger grain size and smaller crystallite size. 


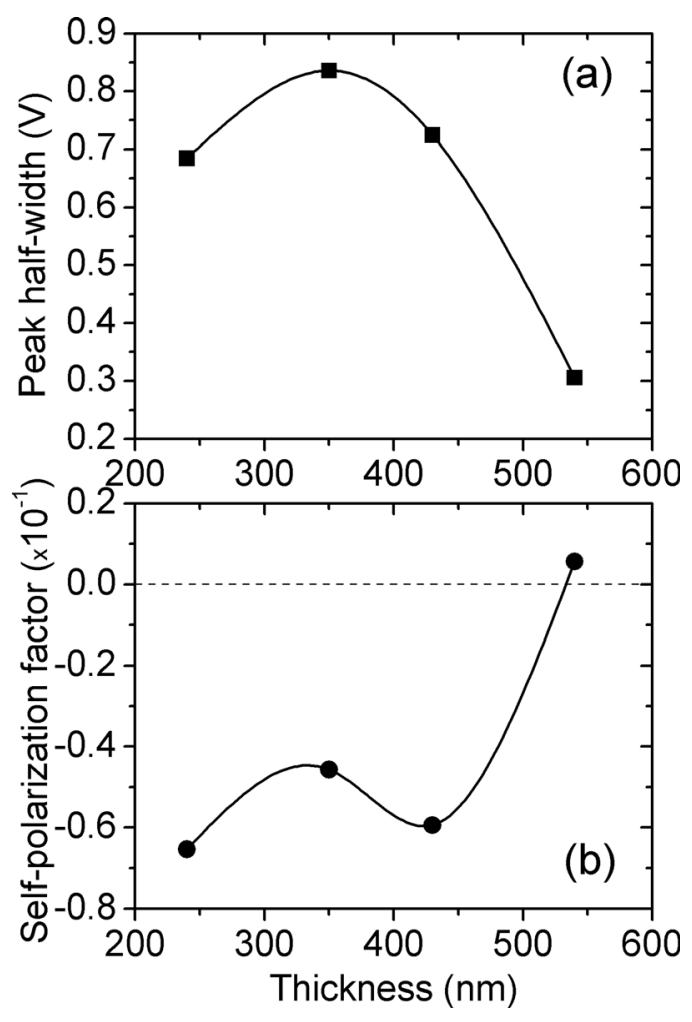

FIG. 5. (a) Thickness dependence of peak half-width of piezohistograms in Fig. 4(b). (b) Thickness dependence of self-polarization factor (see text) of PLZT thin films. Lines in both curves are drawn as a guide to the eye.

The asymmetries observed in the piezohistograms of Fig. 4(b) are other points to consider. In this figure, the $d_{33}$ peaks shift slightly to negative voltages for films with 240 , 350 , and $430 \mathrm{~nm}$ in thickness, but the piezohistogram of the thicker film $(540 \mathrm{~nm}$ thick) is almost symmetrical around $0 \mathrm{~V}$ $\left(d_{33}\right.$ peak $\left.\sim 5.7 \times 10^{-3} \mathrm{~V}\right)$. These asymmetries may be due to instrumental effects or to an imprint effect in the films. First, let us punctuate possible instrumental effects associated with tip geometry, to electrostatic effects or to piezoelectric interactions due to tip-sample interactions on the PFM signal. Although we used a metallic spherical tip in our measurements, it was demonstrated that PFM width signal across a sharp ferroelectric domain wall is linear with tip contact radius and that the magnitude of the effective piezoelectric coefficient $d_{33}$ is independent on the tip radius. ${ }^{20}$ Other reports demonstrate that asymmetries on the local hysteresis loops can be described in terms of a capacitive contribution $\left(\propto V_{a c} / k\right)$ of the cantilever and a piezoelectric contribution $\left(d_{33} V_{a c}\right)$, where $k$ is the spring constant of the cantilever. $^{21}$ In our study, we used stiff cantilevers interacting with film surface only. Consequently, the capacitive contribution is expected to be minimal, and the piezoelectric part dominates the PFM signal without injection effect due to the potential barrier between the tip and film surface. Thus, we expect no direct influence of bottom electrode on the electrostatic effects, as well no charge injection effect due to the potential barrier between the tip and surface of the film. Based on these assumptions, we excluded tip geometry or electrostatic effects as mechanisms responsible for the asymmetries in piezohistograms in Fig. 4(b).
Excluding instrumental effects, we must consider imprint as possible cause for the small asymmetries observed on the distribution of the piezoresponse signal. The imprint phenomenon, referred sometimes as a self-polarization effect, is often observed in ferroelectric thin films and is characterized by asymmetries on the hysteresis loops at macroscale and nanoscale due to the presence of an internal built-in electric field. Considering the distribution of piezoresponse signal, selfpolarization can be evaluated by the $\beta$ factor according the following equation: ${ }^{20}$

$$
\beta=\frac{\int_{0}^{+\infty} N(v) d v-\int_{-\infty}^{0} N(v) d v}{\int_{-\infty}^{+\infty} N(v) d v}
$$

Eq. (1) reflects the difference between all positive and all negative domain states divided by all domain states in the studied range. Thus, Fig. 5(b) plots the behavior of the selfpolarization $\beta$ factor as a function of thickness to infer the origin of asymmetries in the piezohistograms.

The self-polarization factors in Fig. 5(b) slightly shifting to negative values for thinner films suggest a predominance of "negative" domains over the oppositely oriented "positive" domains, indicating a polarization head directed toward the bulk of the film. For thicker film, the selfpolarization factor is almost zero. Weak self-polarization in thicker film suggests a predominance of domains toward the free surface of the film. Asymmetries observed in slim hysteresis loops of the PLZT film with $540 \mathrm{~nm}$ in thickness (not shown here) reveal that $\Delta \mathrm{P}_{\mathrm{r}}=P_{r}^{+}-P_{r}^{-}=+0.7 \mu \mathrm{C} / \mathrm{cm}^{2}$. These results mean that for both local and macroscale cases, the net remanent polarization $\left(\Delta \mathrm{P}_{\mathrm{r}}\right)$ is positive. Despite different magnitudes, the positive liquid remanent polarization at macroscale agrees with the positive local self-polarization observed in Fig. 5(b) for the thicker film (540 nm thick). In addition, although macroscale $\mathrm{P}-\mathrm{E}$ hysteresis loops were not obtained for thinner films, the self-polarization factor shifting to negative values in piezohistograms of Fig. 5(b) for thinner films (larger grain sizes) indicates the existence of a build-in electric field toward the bottom film-substrate interface, while for thicker film (smaller grain sizes), a small build-in electric field toward film free surface.

The origin of the self-polarization in ferroelectric thin films has been discussed in terms of different mechanisms such as Schottky barriers, ${ }^{22}$ lead vacancies, ${ }^{23}$ mechanical coupling between the film and the substrate, ${ }^{24}$ formation temperature of the ferroelectric phase, ${ }^{25}$ and some others. When the top and bottom electrodes of a metal/ferroelectric/ metal configuration are made of different materials, trapped charges appear near the bottom ferroelectric-electrode interface leading to a build-in electric field responsible for the self-polarization. Lead and oxygen vacancies are complex defects in $\mathrm{Pb}$-based thin films that may be also responsible by localized build-in electric field in the ferroelectric film responsible by an imprint effect. Such defects appear in these materials due to several factors such as $\mathrm{PbO}$ loss and oxygen pressure during the synthesis or donor/acceptor impurities introduced in the perovskite $\mathrm{ABO}_{3}$ structure. ${ }^{24}$ Additionally, 
strain gradients along the film depth sometimes appear in epitaxial films due to a perfect match between lattice parameters of film-substrate. This strain gradient is another mechanism responsible for the self-polarization. On the other hand, different thermal expansion coefficients between filmsubstrate may originate a compressive or tensile stress on cooling after the film crystallization that will produce a selfpolarized state.

The space-charge field is another point to consider as possible origin of build-in electric field in PLZT thin films. In literature, it was observed that the space-charge field increases with decreasing grain size and increasing porosity in PLZT ceramics. ${ }^{26}$ In the present work, the selfpolarization factors shifting to negative values for thinner films suggest that build-in electric field increases with increasing grain size. Although porosity is relatively high in thin films prepared by the polymeric chemical method used in the present work, if compared to thin films prepared by physical methods such as rf-sputtering or laser ablation, it is improbable that space-charge is the dominant mechanism responsible for the build-in electric field in PLZT films studied in the present work. Despite different models are used to explain the mechanisms of the built-in electric field in ferroelectric films, ${ }^{27}$ the nature of this effect is still under discussion and naturally depends on each particular system studied.

Considering the random oriented PLZT films studied in this work and the expected low thermal expansion coefficients between the film and the substrate, we exclude strain gradients along the film depth and compressive/tensile stress as mechanisms of self-polarization in these films.

Although recent studies on the thickness dependence of self-polarization in PZT thin films ${ }^{28}$ prepared by the same chemical method used in the present work have excluded Schottky barriers and mechanical coupling near the filmsubstrate interface and assumed that complex defects are the probable mechanisms responsible by self-polarization, ${ }^{29}$ the situation observed in the present work for PLZT films is different. In other words, while the self-polarization in PZT films occurs in the bulk, our results indicate the Schottky barriers close to the bottom film-substrate interface seems the dominant mechanism responsible for the self-polarization in PLZT film. The increase in self-polarization with decreasing film thickness in the present work first suggests that the alignment of ferroelectric domains may occur near the film-electrode interface. In this context, Schottky barriers near the filmsubstrate are likely responsible for the build-in electric field in the studied PLZT films. However, further experiments are in progress to clarify the mechanism of self-polarization in PLZT films studied in this work.

Now, we conduct a quantitative analysis of nanodomain structure of studied PLZT films from autocorrelation images obtained by applying the transformation $C\left(r_{1}, r_{2}\right)=\sum_{x, y}$ $D(x, y) D\left(x+r_{1}, y+r_{2}\right)$ on the piezoresponse images in Fig. 3(b). In this equation, $D(x, y)$ is the piezoresponse signal and $C\left(r_{1}, r_{2}\right)$ is the autocorrelation function that describes some regularity of the nanodomain structure in a specific direction on the film surface. Then, the short-range correlations can be described by the equation $C(r)=\sigma^{2} \exp \left[-(r / \xi)^{2 h}\right]$, where $\sigma$ is a pre-exponential factor, $\xi$ is the correlation length and $h$ $(0<h<1)$ is a parameter related to the roughness of a "polarization interface." 30 Based on the autocorrelation images (not shown here), the autocorrelation $C(r)$ at small $r$ were recorded for PLZT thin films with different thicknesses and plotted in Fig. 6(a) with its corresponding best fits.

The thickness dependence of the correlation length $\xi$ obtained from fits is shown in Fig. 6(b). The larger correlation length $\xi$ in the present study and its maximum value observed for the film with $350 \mathrm{~nm}$ in thickness are two aspects to punctuate. The correlation lengths obtained in this work are about two to three times larger than those reported in literature for PLZT ceramics at the same composition ${ }^{31}$ and comparable to larger values observed for $\mathrm{Ba}(\mathrm{Zr}, \mathrm{Ti}) \mathrm{O}_{3}-(\mathrm{Ba}, \mathrm{Ca}) \mathrm{TiO}_{3}$ (BZT-BCT) thin films, ${ }^{32}$ while the typical correlation lengths observed for relaxor thin films are $\sim 80 \mathrm{~nm} .{ }^{33}$ Although it remains unclear the relationship between correlation length and its real value at macroscale, we assume that $\xi$ is directly related to the average size of polar nanoregions (PNR) in relaxors $^{34}$ and thus it can be used to measure the polarization disorder on the surface of the relaxor films. The great similarity between results shown in Figs. 4, 5(a), and 6(b) suggests a correspondence between grain size and correlation length. Based on these results, there was smaller correlation length in small grains and higher correlation length for large grains. These results are consistent with previous report on the grain size effect in PLZT ceramics, which demonstrated larger correlation length for larger grain size. ${ }^{32}$

Large correlation lengths obtained in the present work suggests larger PNRs in the studied PLZT films. In literature, large PNRs observed in BZT-BCT films $(\xi \sim 151 \mathrm{~nm})$ have been attributed to stronger polar correlations due to the seeded layer grown between the film-substrate such that the

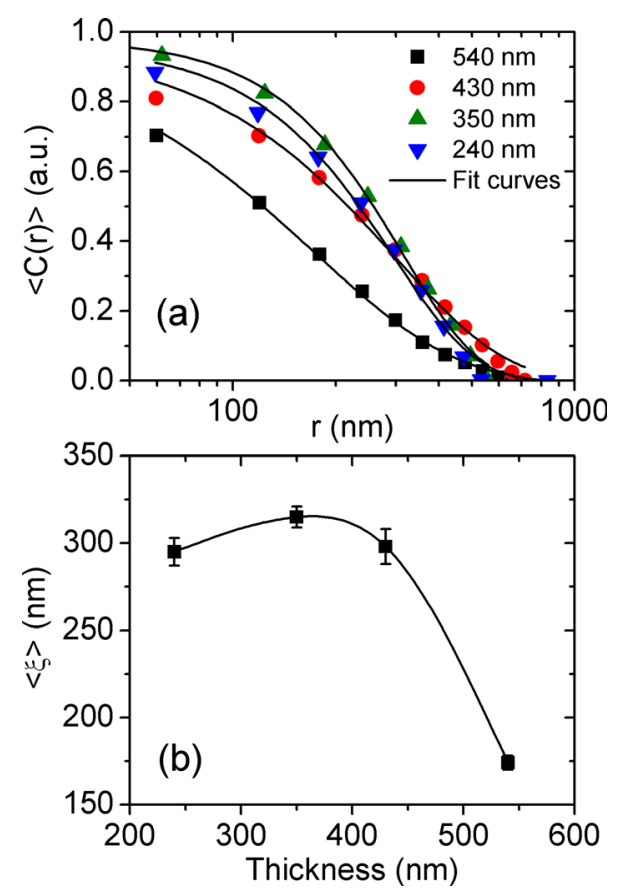

FIG. 6. (a) Distance dependence of autocorrelation function $C(r)$ and (b) Thickness dependence of correlation length $\xi$ of PLZT films. Lines in (a) are best fits of $C(r)$ while in (b) lines are drawn as a guide to the eye. 
seed layer is the responsible for nucleation and growth of large nanodomains. ${ }^{33}$ In our PLZT thin films, however, the large PNRs are certainly due to other mechanisms. The $\mathrm{La}^{3+}$ substitution on the A site of PZT system breaks the translational symmetry of the lattice, leading to a disorder responsible for the condensation of randomly oriented polar nanodomains below the Burns temperature. ${ }^{35}$ The feature of the nanodomains at room temperature depends on the thermal history. On cooling in the absence of biasing electric field, the domains can become largely sufficient to produce macrodomains permeating the sample, or domains can freeze in nanodomains with random orientation. The first assumption leads to a cooperative ferroelectric state while the second one leads to a relaxor state with nanodomains randomly oriented. In addition, in the presence of a biasing electric field, it is expected an alignment of domains followed by an increase in their correlation length on cooling leading to a nano-to-macrodomain transition in PLZT. ${ }^{35}$ The increase observed in correlation length with increasing of selfpolarization for thinner films (larger grains) is a strong evidence of the build-in electric field effects on the alignment of macrodomains in the grains.

\section{CONCLUSIONS}

In summary, the thickness and grain size dependences of structural and piezoresponse properties at nanoscale of PLZT thin films were investigated. The thinnest films show a slight (100)-orientation tendency while the thicker film has a structure with random orientation, and their crystallite size and microstrain increases almost linearly with increasing thickness. An imprint effect characterized by a selfpolarization with thickness dependence was observed in the studied films. The self-polarization increases for thinner films and tends to disappear for the thicker one. This behavior excluded mechanisms in the bulk and suggests that Schottky barriers near the film-substrate are likely responsible by build-in electric field in the films. Under this build-in field, smaller crystallite sizes in larger grains increases the polarization directions in coexistence leading to an alignment of macrodomains in the grains of the thinner films. The increase in correlation length with the increase in selfpolarization for thinner films is a strong evidence of this build-in field effect.

\section{ACKNOWLEDGMENTS}

We would like to express our gratitude to the Brazilian agencies CNPq (Research Grant No. 304604/2015-1 and Project No. 400677/2014-8), CAPES and FAPESP for financial support. The research was made possible in part by Russian Foundation for Basic Research (Grant No. 16-02-00821-a). The work was supported by Government of the Russian Federation (Act 211, Agreement No. 02.A03.21.0006). Part of this work was developed in the scope of Project CICECO-Aveiro Institute of Materials (Ref. FCT UID/CTM/50011/2013), financed by national funds through the FCT/MEC and, when applicable, cofinanced by FEDER under the PT2020 Partnership Agreement.

${ }^{1}$ G. H. Haertling, "Electro-optic ceramics and devices," in Electronic Ceramics edited by L. M. Levinson (Marcel Dekker, New York, NY, USA, 1988).

${ }^{2}$ G. H. Haertling, Ferroelectrics 75, 25 (1987).

${ }^{3}$ C. S. Lynch, Acta Mater. 44, 4137 (1996).

${ }^{4}$ G. H. Haertling, J. Am. Ceram. Soc. 82, 797 (1999).

${ }^{5}$ R. Thapliya, Y. Okano, and S. Nakamura, J. Lightwave Technol. 21, 1820 (2003).

${ }^{6}$ Z. Q. Hu, B. H. Ma, S. S. Liu, M. Narayanan, and U. Balachandran, Ceram. Int. 40, 557 (2014).

${ }^{7}$ S. C. Lai, K. Yao, Y. F. Chen, L. Zhang, and Y. F. Lim, IEEE Electron Devices Lett. 34, 1427 (2013).

${ }^{8}$ E. B. Araujo, B. O. Nahime, M. Melo, F. Dinelli, F. Tantussi, P. Baschieri, F. Fuso, and M. Allegrini, Mater. Res. Bull. 61, 26 (2015).

${ }^{9}$ H. M. Rietveld, Acta Crystallogr. 22, 151 (1967).

${ }^{10}$ B. H. Toby, J. Appl. Cryst. 34, 210 (2001).

${ }^{11}$ P. Thompson, D. E. Cox, and J. B. Hastings, J. Appl. Crystallogr. 20, 79 (1987).

${ }^{12}$ L. B. McCusker, R. B. Von Dreele, D. E. Cox, D. Louër, and P. Scardi, J. Appl. Cryst. 32, 36 (1999).

${ }^{13}$ N. Balke, I. Bdikin, S. V. Kalinin, and A. L. Kholkin, J. Am. Ceram. Soc. 92, 1629 (2009).

${ }^{14}$ V. V. Efimov, E. A. Efimova, K. Iakoubovskii, S. Khasanovc, D. I. Kochubey, V. V. Kriventsov, A. Kuzmin, B. N. Mavrin, M. Sakharov, V. Sikolenko, A. N. Shmakov, and S. I. Tiutiunnikov, J. Phys. Chem. Solids 67, 2007 (2006).

${ }^{15}$ X. Y. Wen, J. Yu, Y. B. Wang, W. L. Zhou, and J. X. Gao, J. Appl. Phys. 108, 114103 (2010).

${ }^{16}$ G. Leclerc, B. Domengès, G. Poullain, and R. Bouregba, Appl. Surf. Sci. 253, 1143 (2006).

${ }^{17}$ S. Q. Zhang, L. D. Wang, W. L. Li, N. Li, and W. D. Fei, J. Alloys Compd. 509, 2976 (2011).

${ }^{18}$ A. Ferri, S. Saitzek, A. Da Costa, R. Desfeux, G. Leclerc, R. Bouregba, and G. Poullain, Surf. Sci. 602, 1987 (2008).

${ }^{19}$ A. Wu, P. M. Vilarinho, V. V. Shvartsman, G. Suchaneck, and A. L. Kholkin, Nanotechnology 16, 2587 (2005).

${ }^{20}$ L. Tian, A. Vasudevarao, A. N. Morozovska, E. A. Eliseev, S. V. Kalinin, and V. Gopalan, J. Appl. Phys. 104, 074110 (2008).

${ }^{21}$ S. Hong, J. Woo, H. Shin, J. U. Jeon, Y. E. Pak, E. L. Colla, N. Setter, E. Kim, and K. No, J. Appl. Phys. 89, 1377 (2001).

${ }^{22}$ A. L. Kholkin, K. G. Brooks, D. V. Taylor, S. Hiboux, and N. Setter, Integr. Ferroelectr. 22, 525 (1998).

${ }^{23}$ V. P. Afanasjev, A. A. Petrov, I. P. Pronin, E. A. Tarakanov, E. Ju. Kaptelov, and J. Graul, J. Phys.: Condens. Matter 13, 8755 (2001).

${ }^{24}$ A. Gruverman, B. J. Rodriguez, A. I. Kingon, R. J. Nemanich, A. K. Tagantsev, J. S. Cross, and M. Tsukada, Appl. Phys. Lett. 83, 728 (2003).

${ }^{25}$ V. P. Afanasjev, I. P. Pronin, and A. L. Kholkin, Phys. Solid State 48, 1214 (2006).

${ }^{26}$ K. Okazaki and K. Nagata, J. Am. Ceram. Soc. 56, 82 (1973).

${ }^{27}$ A. K. Tagantsev and G. Gerra, J. Appl. Phys. 100, 051607 (2006).

${ }^{28}$ E. B. Araujo, E. C. Lima, I. K. Bdikin, and A. L. Kholkin, J. Appl. Phys. 113, 187206 (2013).

${ }^{29}$ E. B. Araujo, E. C. Lima, I. K. Bdikin, and A. L. Kholkin, Ferroelectrics 498, 18 (2016).

${ }^{30}$ R. C. Munoz, G. Vidal, M. Mulsow, J. G. Lisoni, C. Arenas, A. Concha, F. Mora, and R. Espejo, Phys. Rev. B 62, 4686 (2000).

${ }^{31}$ D. A. Kiselev, I. K. Bdikin, E. K. Selezneva, K. Bormanis, A. Sternberg, and A. L. Kholkin, J. Phys. D: Appl. Phys. 40, 7109 (2007).

${ }^{32}$ W. L. Li, T. D. Zhang, Y. F. Hou, Y. Zhao, D. Xu, W. P. Cao, and W. D. Fei, RSC Adv. 4, 56933 (2014).

${ }^{33}$ V. V. Shvartsman, B. Dkhil, and A. L. Kholkin, Annu. Rev. Mater. Res. 43, 423 (2013).

${ }^{34}$ W. Kleemann, J. Mater. Sci. 41, 129 (2006).

${ }^{35}$ G. A. Samara, J. Phys.: Condens. Matter 15, R367 (2003). 